\title{
Charge calibration of DRZ scintillation phosphor screens
}

\author{
J.-P. Schwinkendorf, ${ }^{a, b}$ S. Bohlen, ${ }^{a, b}$ J. P. Couperus Cabadağ ${ }^{c}$ H. Ding, ${ }^{d, e}$ A. Irman, ${ }^{c} \mathbf{S}$. \\ Karsch, $^{d, e}$ A. Köhler, ${ }^{c, f}$ J. M. Krämer, ${ }^{c, f}$ T. Kurz, ${ }^{c, d, f}$ S. Kuschel, ${ }^{g, h}$ J. Osterhoff, ${ }^{a}$ L. F. \\ Schaper, ${ }^{a}$ D. Schinkel, ${ }^{g, h}$ U. Schramm, ${ }^{c, d}$ O. Zarini, ${ }^{c, d}$ and R. D’Arcy ${ }^{a, 1}$ \\ a Deutsches Elektronen-Synchrotron DESY, \\ Notkestr. 85, 22607 Hamburg, Germany \\ ${ }^{b}$ Institut für Experimentalphysik, Universität Hamburg, \\ Luruper Chaussee 149, 22761 Hamburg, Germany \\ ${ }^{c}$ Helmholtz-Zentrum Dresden-Rossendorf, \\ Bautzener Landstraße 400, 01328 Dresden, Germany \\ ${ }^{d}$ Ludwig-Maximilians-Universität München, \\ Am Coulombwall 1, 85748 Garching, Germany \\ ${ }^{e}$ Max-Planck-Institut für Quantenoptik, \\ Hans-Kopfermann-Straße 1, 85748 Garching, Germany \\ ${ }^{f}$ Technische Universität Dresden, \\ 01069 Dresden, Germany \\ ${ }^{g}$ Helmholtz-Institut Jena, \\ Fröbelstieg 3, 07743 Jena, Germany \\ ${ }^{h}$ Friedrich-Schiller-Universität Jena, \\ Fürstengraben 1, 07743 Jena, Germany \\ E-mail: richard.darcy@desy.de
}

AвSTRACT: As a basic diagnostic tool, scintillation screens are employed in particle accelerators to detect charged particles. In extension to the recent revision on the calibration of scintillation screens commonly applied in the context of plasma acceleration [T. Kurz et al., Rev. Sci. Instrum. 89 (2018) 093303], here we present the charge calibration of three DRZ screens (Std, Plus, High), which promise to offer similar spatial resolution to other screen types whilst reaching higher conversion efficiencies. The calibration was performed at the Electron Linac for beams with high Brilliance and low Emittance (ELBE) at the Helmholtz-Zentrum Dresden-Rossendorf, which delivers picosecondlong beams of up to $40 \mathrm{MeV}$ energy. Compared to the most sensitive screen, Kodak BioMAX MS, of the aforementioned recent investigation by Kurz et al., the sample with highest yield in this campaign, DRZ High, revealed a 30\% increase in light yield. The detection threshold with these screens was found to be below $10 \mathrm{pC} / \mathrm{mm}^{2}$. For higher charge-densities (several $\mathrm{nC} / \mathrm{mm}^{2}$ ) saturation effects were observed. In contrast to the recent reported work, the DRZ screens were more robust, demonstrating higher durability under the same high level of charge deposition.

Keywords: Wake-field acceleration; Beam-line instrumentation; Scintillators, scintillation and light emission processes; Detector alignment and calibration methods

\footnotetext{
${ }^{1}$ Corresponding author
} 


\section{Contents}

1 Introduction 1

2 Setup 2

3 Results 3

3.1 Absolute charge calibration 3

3.2 Saturation 5

3.3 Degradation 6

4 Conclusion $\quad 7$

\section{Introduction}

With the development of high-power laser systems [2-4] plasma-based wakefield acceleration [5] has evolved into an intense research area. Focusing such a laser into a plasma may nowadays create ultra-fast quasi-monoenergetic electron bunches [6-8] of energies within the $\mathrm{MeV}$ to $\mathrm{GeV}$ range [9-12] and bunch charges of $\mathrm{pC}$ up to the $\mathrm{nC}$ range [13] resulting in high peak currents $[14,15]$. Compared with conventional radio-frequency (RF) accelerators shot-to-shot stability of such schemes can be less consistent. It is, therefore, of special interest to characterise the electron beam properties with a single shot in order to understand and subsequently stabilise this process [16].

For investigation of the electron energy spectrum, a dipole magnet is typically incorporated, which disperses the electron bunch and thus maps the particle energy to an off-axis position, the size of which can extend over several tens of $\mathrm{cm}$ in order to maximise its resolving power. Therefore, electron beams from plasma accelerators can be dispersed to a few $\mathrm{fC} / \mathrm{mm}^{2}$ [17]. Subsequently, the dispersed beam needs to be detected by a charge sensitive device. Most conventional electronicsbased charge diagnostics suffer from the noisy environment of the laser-plasma interaction. In this context, scintillators can be applied as converters. Those made of phosphor gadolinium oxysulfide $\left(\mathrm{Gd}_{2} \mathrm{O}_{2} \mathrm{~S}\right)$, for example - widely used in industrial or medical imaging with X-rays e.g. the trademark LANEX originally supplied by Kodak - have become a standard tool. By emitting photons in the green frequency range of the visible light spectrum, they are advantageous for imaging the scintillator with charged-coupled devices (CCDs). The excited states in the scintillator

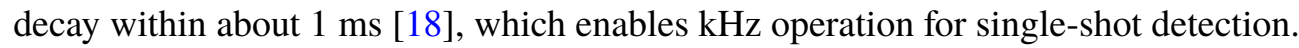

There is a broad range of scintillation screens available with various spatial resolutions, sensitivities, and decay times, for which several investigations and calibrations have already been performed [1, 19-21]. Based on their availability, emission characteristics, and simple application, the $\mathrm{Gd}_{2} \mathrm{O}_{2} \mathrm{~S}$ scintillation screens are common options in the field of plasma acceleration. Besides the broad range of options already in general use, MCI Optonix / Mitsubishi Chemical offers screens 
of this kind with their "DRZ series", which promise to give similar spatial resolutions but at the same time a higher light yield ${ }^{1}$. For these reasons they are of special interest for plasma acceleration requiring a low charge detection threshold.

Due to the developmental nature of the field, screens with a high radiation hardness are also desired in order to withstand occasional high charge (density) shots. Since these screens are produced for the context of X-ray applications a charge calibration and lifetime test is required for their usage within the scope of electron beam diagnostics. For this reason calibration studies of the three scintillation screen types - DRZ Std, DRZ Plus, and DRZ High - have been performed over a wide charge (density) range. Besides the linear response of the screens (section 3.1), saturation (section 3.2), and short- as well as long-term degradation effects (section 3.3) have been studied.

\section{Setup}

The charge calibration measurements were performed at the Electron Linac for beams with high Brilliance and low Emittance (ELBE) linear accelerator (LINAC) at the Helmholtz-Zentrum DresdenRossendorf (HZDR). The machine is capable of delivering pulse trains of variable length at a repetition rate of $1 \mathrm{kHz}$ with a micro bunch repetition rate of $13 \mathrm{MHz}$. The single bunches have pulse durations of $2 \mathrm{ps}$, tuneable charges of up to $80 \mathrm{pC}$, and maximum energies of $40 \mathrm{MeV}$.

For experimentation with lower charges down to $5 \mathrm{pC}$ the LINAC delivered single electron bunches with an energy of $23 \mathrm{MeV}$ at a repetition rate of $1 \mathrm{~Hz}$. For the higher charges of $50 \mathrm{pC}$ up to $50 \mathrm{nC}$ the accelerator was operated in a bunch train mode with multiple pulses in one train of tuneable length running with a temporal spacing of $77 \mathrm{~ns}$ between the single pulses in the train. For the maximum investigated charge a train is constructed with up to 1000 single bunches, each with a charge of $50 \mathrm{pC}$. In this case the irradiation time of the screen rises to about $0.1 \mathrm{~ms}$ which is comparable to, but still less than, the lifetime of the excited scintillation states (on the order of a $\mathrm{ms}$ ). The electron beam is focused by magnetic quadrupoles to a 1/e-beam size of approx. $3 \mathrm{~mm}^{2}$ resulting in a peak charge density of up to approx. $15 \mathrm{nC} / \mathrm{mm}^{2}$. The beam area was deduced from the screen signal by taking lineouts through the signal centre and applying Gaussian fits for each shot.

Figure 1 depicts the calibration setup. The experimental vacuum vessel was connected directly to the accelerator without any window in between, thus mitigating background from Bremsstrahlung. After acceleration, the electrons passed through an integrating current transformer (ICT-082-0705:1-VAC, Bergoz Instruments, France) in order to measure the beam charge. The charge measurement was calibrated [1] yielding an error of 5\% [22]. Afterwards, the screens mounted in a rotatable target wheel were irradiated. Simulation and measurements show that the energy deposition per electron into the phosphor layer is almost independent for electron energies above 3 $\mathrm{MeV}$ [19, 23-25]. Hence, this calibration is applicable for all experiments reaching this threshold. The light-emission distribution of phosphor screens approximately follows Lambert's cosine law [26]. Thus the highest density of green photons are emitted perpendicular to the screen surface. Therefore, the wheel was positioned at $(22 \pm 1)^{\circ}$ with respect to the beam propagation axis to keep the subsequent pick-up optics out of the electron beam path and to minimise possible background.

${ }^{1}$ http://www.mcio.com/Products/drz-screens. aspx 


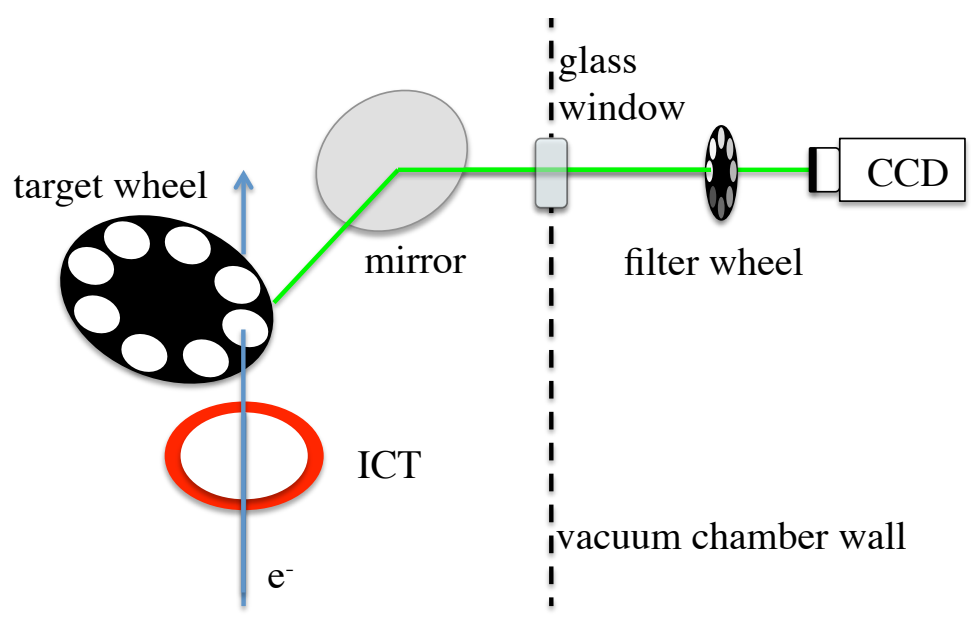

Figure 1. Charge calibration setup: The electrons $\left(\mathrm{e}^{-}\right)$from the ELBE linear accelerator are focused through an ICT, measuring the bunch charge, onto the scintillation screens mounted in a rotatable target wheel. The wheel is positioned at $22^{\circ}$ with respect to the electron beam path. Thus, the subsequent pick-up mirror is not interfering with the electrons and the scintillation signal can be optically imaged perpendicular to the screen's surface through the vacuum chamber window and a filter wheel onto a CCD camera.

The emitted light was reflected by a silver mirror (Thorlabs, PF20-03-P01) placed at $(34 \pm 1)^{\circ}$ out of the vacuum chamber such that the photon-collecting 12-bit CCD camera (Basler acA1300-30gm) - equipped with an ND 1.0 filter (90\% attenuation) and a Pentax TV zoom lens (12.5-75 mm, 1:1.8) mounted via a Thorlabs c-mount ring - orthogonally images the screen surface to maximise the output signal. In front of the camera another filter wheel was positioned with filters ranging from ND 0.5 to ND 4.0 to increase the dynamic range. All ND filters were calibrated separately. Background pictures including the dark current of the accelerator were recorded and then subtracted accordingly.

The imaging system collected light in a solid angle of $\Omega=2.9 \cdot 10^{-3} \mathrm{sr} \pm 2.5 \%$ such that lateral signal variations owing to the Lambertian emission characteristics may be ignored. The reflection/transmission of the mirror $t_{M}$, the chamber window $t_{W}$ and camera objective $t_{O}$ as well as the quantum-efficiency $\varepsilon_{Q E}(\mathrm{QE})$ and digital conversion of the camera result in an efficiency of $T=0.253 \pm 6 \%$.

It should be noted that the data was stored in an 8-bit stream with any associated pixel errors propagated for data analysis. Furthermore, for the low charges below $50 \mathrm{pC}$ the camera was running in a non-linear amplification mode. Therefore, for charges below $50 \mathrm{pC}$ the data is not included in the calibration analysis but still showed the detection capability of the screens in the corresponding charge range down to the $1 \mathrm{pC}$ level.

\section{Results}

\subsection{Absolute charge calibration}

To quantify the photon emission of the scintillation screens a region-of-interest (ROI) was defined in the acquired images to minimise the background noise. An example image of an electron 


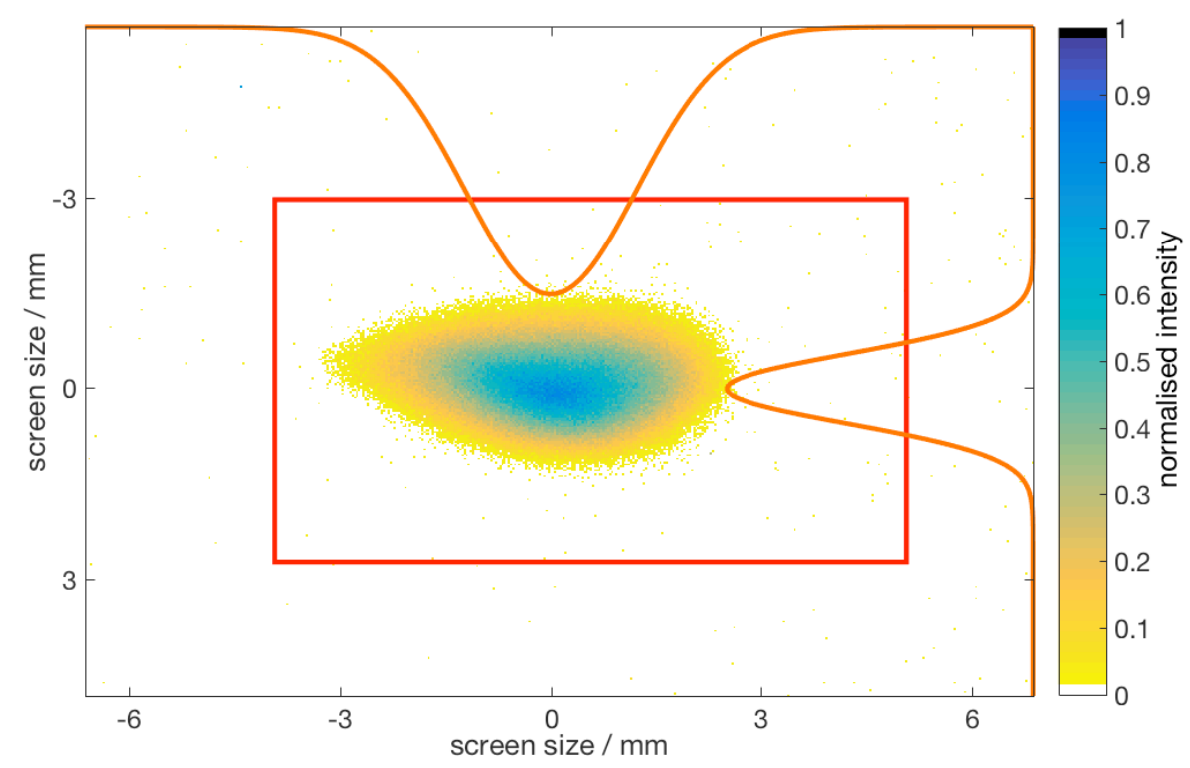

Figure 2. Example image of an effective $200 \mathrm{pC}$ electron bunch impinging on the scintillation screen recorded by the CCD sensor. The red rectangle marks the region of interest (ROI) for the calibration data analysis. The two inset curves indicate the horizontal and vertical profile (Gaussian fits of the line-out through the electron bunch profile intensity peak).

beam interacting with a screen can be seen in Figure 2. The CCD counts within the ROI were integrated and background corrected for e.g. thermal noise, bremsstrahlung, and dark current of the accelerator. The absolute photon yield $Y_{S}$ then results from the total number of photons emitted by the scintillator $N_{p h}$ into a solid angle of a steradian per incident electron beam charge $Q_{e}$,

$$
Y_{S}=\frac{N_{p h}}{Q_{e}}=\frac{N_{c} \cos \left(22^{\circ}\right)}{T \Omega Q_{e}}
$$

The photon number $N_{p h}$ is derived from the integrated and background-corrected CCD counts $N_{c}$ within the ROI divided by the optical light collection efficiency and transmission $T=t_{M} t_{W} t_{O} \varepsilon_{Q E}$. The transmission efficiency of the ND filters is included. The effective collection angle is denoted by $\Omega$. The cosine-term accounts for the tilted screen position with respect to the impinging electron beam path and thus for the increased interaction length inside the active layer of the scintillator resulting in a higher light output.

The response of the different scintillation screens (photons/sr) against the impinging charge measured by the ICT is shown in Figure 3. In the $\mathrm{pC}$ charge regime all screens show a linear behaviour whereas for higher charges a drop-off is observable mainly due to saturation effects (cf. section 3.2). Therefore, this higher charge region was excluded from the absolute charge calibration (cf. grey vertical line in Fig. 3).

For each charge 60 shots were captured. The shots were analysed as described and the statistical and systematic errors propagated. Finally, the values were averaged by weighted mean depending on their statistical errors. The resulting absolute charge calibration (photons/sr/pC) for the different screens is indicated in Figure 3 as solid lines and can also be found in Table 1. These results for 


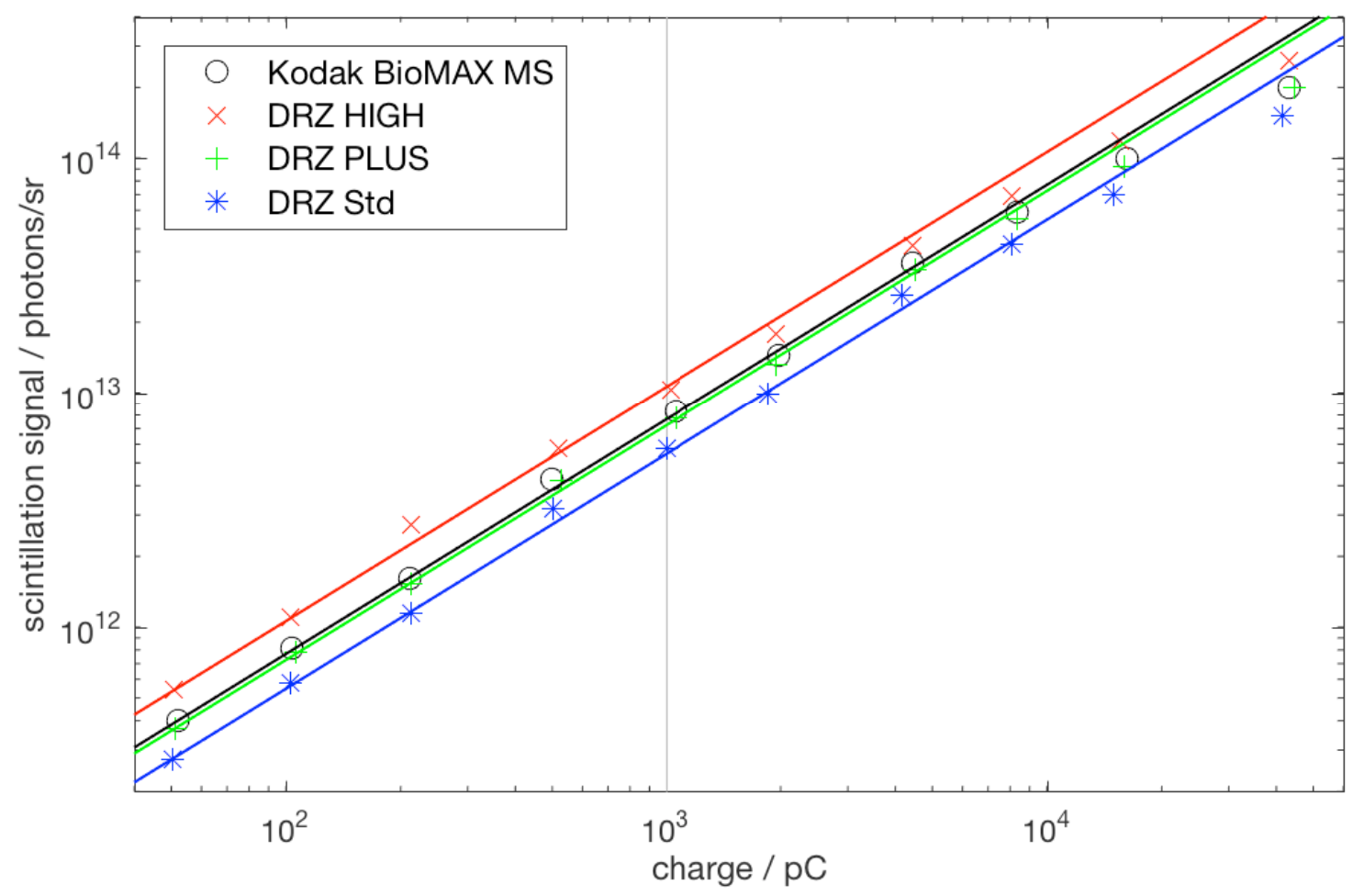

Figure 3. Scintillation response of the three DRZ screens (High, Plus and Std as red cross, green plus and blue asterisk, respectively) as well as Kodak BioMAX MS with black circles for comparison. In the pC range (up to the grey vertical line) the signal of all screens increases linearly whereas with raising charge in the $\mathrm{nC}$ regime a signal drop-off is observable due to saturation. The solid lines depict the result of the charge calibration.

each screen are consistent with the values given by the manufacturer to within the error range. For comparison Kodak BioMAX MS, the most sensitive screen from the recent study [1], was investigated in a similar manner. Its light yield agrees well with the former obtained result and is comparable to that of DRZ Plus. DRZ High has a 30\% higher yield representing the most sensitive screen. It is also important to point out that the result for DRZ High is consistent with that of ref. [25] within errors.

Concerning the error it should be mentioned that it is dominated by the systematic errors. Furthermore, for all charge steps above $100 \mathrm{pC}$ reference shots at $100 \mathrm{pC}$ were taken at intervals between each step to check for damage of the screens (cf. section 3.3). A re-scaling was applied to compensate for degradation effects.

\subsection{Saturation}

With increasing charge the conversion efficiency into photons is reduced based on the saturation in the active layer of the scintillator. This can be seen in Figure 3 and was also observable through a flattening of the beam profile when the camera was not saturated. Birks' law [27] describes the 
Table 1. Charge calibration results including saturation measures with the fit parameter B from Birks' law and the corresponding saturation threshold $\rho_{\text {sat }} @ 95 \%$ at which the light yield is decreased by $5 \%$.

\begin{tabular}{|l||c|c|c|}
\hline screen & $\begin{array}{c}\text { light-yield calibration } \\
\left(10^{9} \text { photons/sr/pC }\right)\end{array}$ & $\begin{array}{c}\mathrm{B} \\
\left(\mathrm{mm}^{2} / \mu \mathrm{C}\right)\end{array}$ & $\begin{array}{c}\rho_{\text {sat }} @ 95 \% \\
\left(\mathrm{nC} / \mathrm{mm}^{2}\right)\end{array}$ \\
\hline \hline DRZ High & $10.6 \pm 3.63$ & $66.3 \pm 4.9$ & $0.79 \pm 0.06$ \\
\hline DRZ Plus & $7.26 \pm 2.42$ & $47.3 \pm 6.0$ & $1.11 \pm 0.14$ \\
\hline DRZ Std & $5.47 \pm 1.87$ & $38.6 \pm 5.8$ & $1.36 \pm 0.20$ \\
\hline Kodak BioMAX MS & $7.61 \pm 2.86$ & $54.6 \pm 5.2$ & $0.96 \pm 0.09$ \\
\hline
\end{tabular}

saturation behaviour of scintillators and can be utilised to fit the response curve

$$
\rho_{\text {scint }}=\frac{\rho_{I C T}}{1+B \cdot \rho_{I C T}},
$$

where the linearity is broken by the fit parameter $B$, and $\rho_{I C T}$ and $\rho_{\text {scint }}$ are the areal charge density measured by the ICT and the charge determined by the scintillator, respectively. Both are normalised to the beam area obtained from the screen (cf. section 2). A saturation threshold $\rho_{\text {sat }}$ is defined as the point at which the effect of saturation results in a drop of light output below $95 \%$ of the unsaturated maximum (cf. Table 1). Figure 4 illustrates this circumstance for DRZ High. The solid black line represents a linear correlation of $\rho_{\text {scint }}$ and $\rho_{I C T}$ in the unrealistic case of zero saturation. The red dashed line shows the fit of the data.

\subsection{Degradation}

In addition to saturation, electron beam-induced degradation was observed. To distinguish between these two effects, $100 \mathrm{pC}$ reference measurements were taken after each increase of the bunch charge during the calibration and were used for re-scaling to decouple the issue of saturation and degradation. During the change of the charge settings the screens were given a few minutes to recover. Throughout the investigation a continuous reduction in light yield of up to about $15 \%$ was observed, similar to other scintillation screens [1].

For further investigation, additional long-term stability measurements independent from the charge calibration were performed. Two long runs were investigated with the DRZ High screen: irradiating the screen with either a) a single $50 \mathrm{pC}$ bunch every second for $1.5 \mathrm{hrs}$, or b) three subsequent bunches in a train with an overall nominal charge of $150 \mathrm{pC}$ every second for $0.5 \mathrm{hrs}$. The 1/e bunch area was approx. $0.9 \mathrm{~mm}^{2}$, such that the total accumulated charge was the same for the two long runs, between which there was a break of more than two hours. Figure 5 shows the result of this study, indicating a decrease in scintillation signal over time suggesting an aging of the screen during use. Fitting the results with an exponential decay gave decay constants of $77.5 \mathrm{nC} / \mathrm{mm}^{2} \pm 6.0 \%$ and $60.0 \mathrm{nC} / \mathrm{mm}^{2} \pm 6.9 \%$ for the 50 and $150 \mathrm{pC}$ runs, respectively. This indicates that the signal loss is charge density-dependent since the higher charge density led to a stronger drop. Furthermore, the degeneration over a long timescale (compared to the repetition rate) is not constant with time in contrast to the shorter timescale investigation of the $100 \mathrm{pC}$ reference shots, where the drop-off appeared linear.

The degradation seems to be permanent. During the break of several hours between the two long runs the screen appeared to have not recovered. The final scintillation signal of the $50 \mathrm{pC}$ 


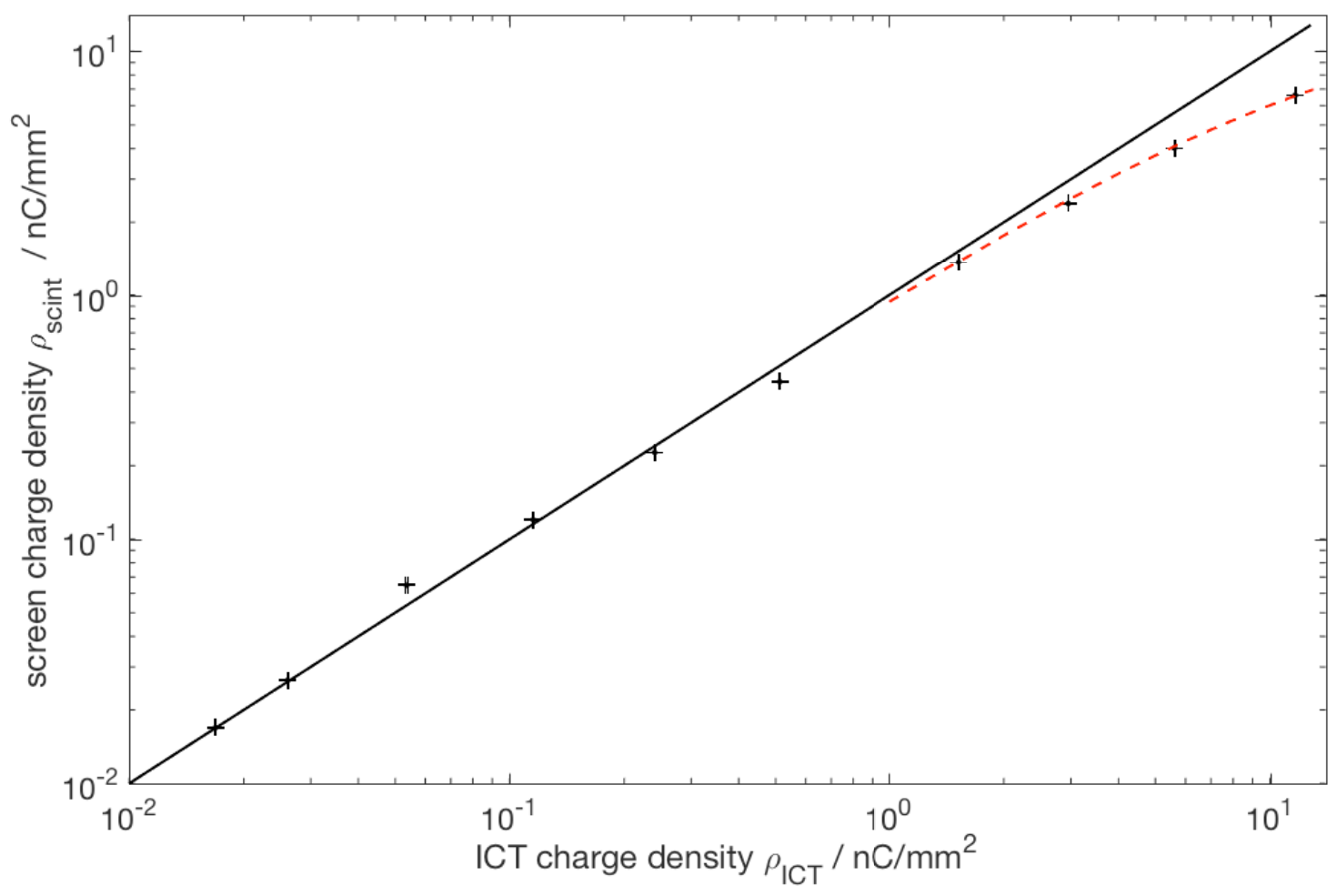

Figure 4. Saturation curve of DRZ High: The charge density $\rho_{\text {scint }}$ derived from the screen against the charge density $\rho_{I C T}$ calculated from the ICT signal and the Gaussian beam profile fitting, fitted with Birks' saturation law. The error bars indicate the statistical errors and are on the order of the data indicating crosses. The solid black line represents the unsaturated case $\left(\rho_{\text {scint }}=\rho_{I C T}\right)$.

run was similar to the starting value of the following $150 \mathrm{pC}$ run divided by the ratio of the bunch charges, whereas in the case of regeneration the equivalent starting value of each long run should have been similar. The improved robustness of the DRZ screens over other types was evident through a lack of blackening, and sometimes destruction, of the screen, as seen in ref. [1].

\section{Conclusion}

The absolute charge calibration and the effect of saturation, as well as short and long term degradation, for three $\mathrm{Gd}_{2} \mathrm{O}_{2} \mathrm{~S}$-based DRZ-type scintillation screens (High, Plus and Std), has been presented. The experimental research was conducted at the ELBE linear accelerator at the HZDR with $23 \mathrm{MeV}$ electron beams of a few ps duration. The screens offer an extension to e.g. LANEX in the portfolio of scintillation screens. In combination with state-of-the-art CCDs, these screens were shown to be capable of detecting charge densities down to a few $\mathrm{pC} / \mathrm{mm}^{2}$ and showed increased robustness for higher charge densities when compared to other screen types. DRZ High showed the highest photon yield of $(10.6 \pm 3.63) \times 10^{9}$ photons $/ \mathrm{sr} / \mathrm{pC}$. This output is higher than the scintillation efficiency of Kodak BioMAX MS - the most sensitive screen investigated thus far - by approximately $30 \%\left((7.61 \pm 2.86) \times 10^{9}\right.$ photons $\left./ \mathrm{sr} / \mathrm{pC}\right)$. 


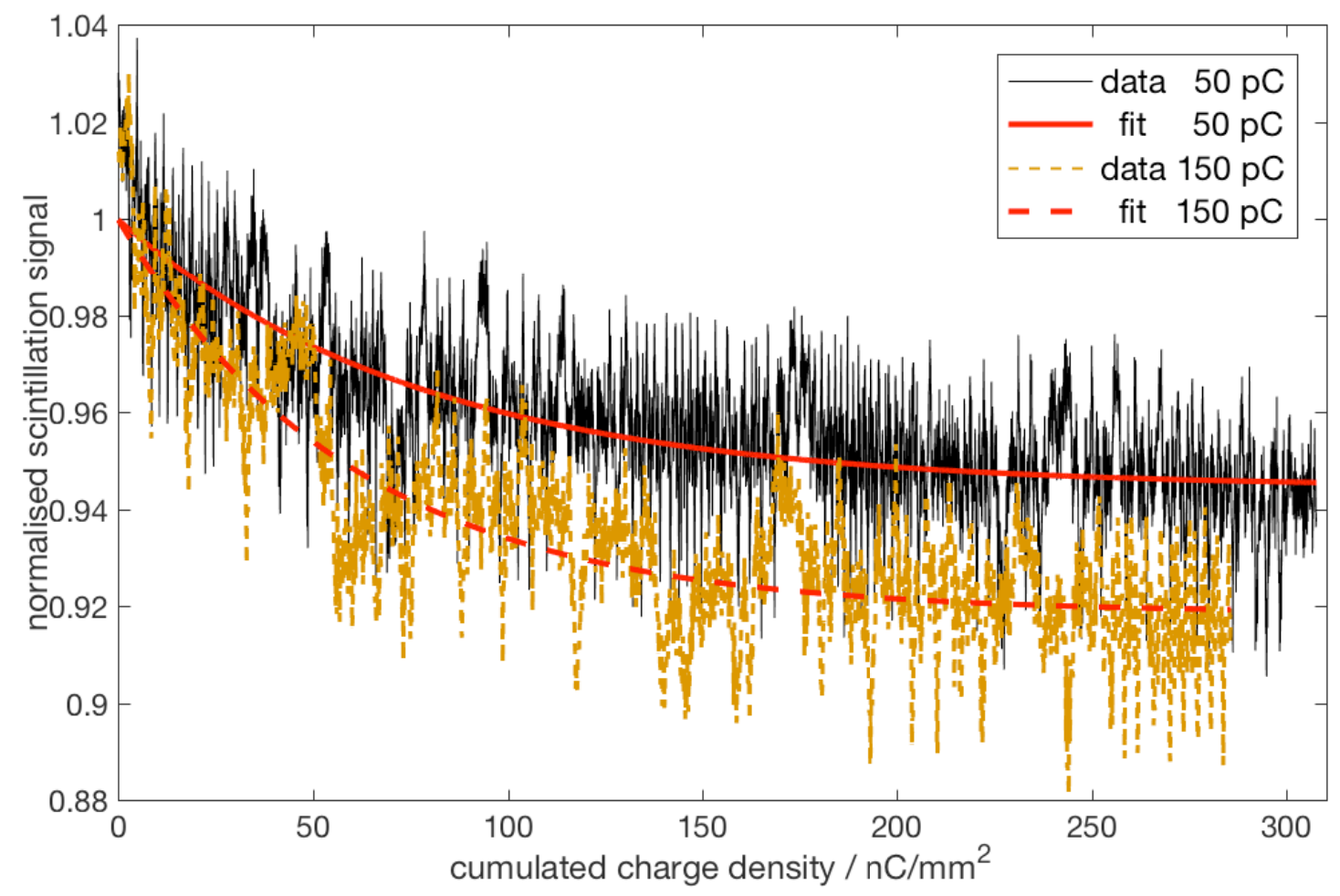

Figure 5. Long run measurement with DRZ High: Scintillation signal against the cumulated charge density with exponential decay fit for two runs of $50 \mathrm{pC}$ bunches over 90 minutes and $150 \mathrm{pC}$ beams over 30 minutes at a repetition rate of $1 \mathrm{~Hz}$. The curves are normalised to the corresponding fit amplitude.

A saturation effect was observed for increasing charge densities on the order of $\mathrm{nC} / \mathrm{mm}^{2}$. The saturation effect gains strength with increasing scintillation efficiency. It has to be accounted for if such high charge densities are reached, which should not be the case for the dispersed beam in an electron spectrometer of a plasma acceleration experiment, for example. The longterm investigation of the DRZ High scintillation screen revealed that aging did occur during the experiment, the magnitude of which depended on the impinging charge density, and did not recover over a break of several hours.

Owing to the unprecedented scintillation efficiency and improved lifetime of DRZ screens, this study has expanded the scope of scintillator usage for e.g. plasma wakefield acceleration, demonstrating the benefits of DRZ over other scintillation screen types.

In combination with the independent calibration standards discussed in ref. [1], the results are portable to be applied within other experimental setups.

\section{Acknowledgments}

This work was supported by the Helmholtz Association ARD research topic, and the Virtual Institute VH-VI-503. 


\section{References}

[1] T. Kurz et al., Calibration and cross-laboratory implementation of scintillating screens for electron bunch charge determination, Rev. Sci. Instrum. 89 (2018) 093303.

[2] E. W. Gaul et al., Demonstration of a 1.1 petawatt laser based on a hybrid optical parametric chirped pulse amplification/mixed Nd:glass amplifier, Appl. Opt. 49 (2010) 1676.

[3] C. Danson, D. Hillier, N. Hopps, and D. Neely, Petawatt class lasers worldwide, High Power Laser Science and Engineering, 3 (2015) E3.

[4] U. Schramm et al., First results with the novel petawatt laser acceleration facility in Dresden, J. Phys.: Conf. Ser. 874 (2017) 012028.

[5] T. Tajima and J. M. Dawson, Laser Electron Accelerator, Phys. Rev. Lett. 43 (1979) 267.

[6] J. Faure et al., A laser-plasma accelerator producing monoenergetic electron beams, Nature 431 (2004) 541.

[7] C. G. R. Geddes et al., High-quality electron beams from a laser wakefield accelerator using plasma-channel guiding, Nature 431 (2004) 538.

[8] S. P. D. Mangles et al., Monoenergetic beams of relativistic electrons from intense laser-plasma interactions, Nature 431 (2004) 535.

[9] W. P. Leemans et al., GeV electron beams from a centimetre-scale accelerator, Nat. Phys. 2 (2006) 696.

[10] X. Wang et al., Quasi-monoenergetic laser-plasma acceleration of electrons to 2 GeV, Nat. Commun. 4 (2013) 1988

[11] H. T. Kim et al., Enhancement of Electron Energy to the Multi-GeV Regime by a Dual-Stage Laser-Wakefield Accelerator Pumped by Petawatt Laser Pulses, Phys. Rev. Lett. 111 (2013) 165002

[12] A. J. Gonsalves et al., Petawatt Laser Guiding and Electron Beam Acceleration to 8 GeV in a Laser-Heated Capillary Discharge Waveguide, Phys. Rev. Lett. 122 (2019) 084801.

[13] J. P. Couperus et al., Demonstration of a beam loaded nanocoulomb-class laser wakefield accelerator, Nat. Commun. 8 (2017) 487.

[14] O. Lundh et al., Few femtosecond, few kiloampere electron bunch produced by a laser-plasma accelerator, Nat. Phys. 7 (2011) 219.

[15] Y. F. Li et al., Generation of 20 kA electron beam from a laser wakefield accelerator, Phys. Plasmas 24 (2017) 023108.

[16] M. C. Downer et al., Diagnostics for plasma-based electron accelerators, Rev. Mod. Phys. 90 (2018) 035002.

[17] E. Adli et al., Acceleration of electrons in the plasma wakefield of a proton bunch, Nature 561 (2018) 363.

[18] R. Morlotti et al., Intrinsic conversion efficiency of X-rays to light in $\mathrm{Gd}_{2} \mathrm{O}_{2} \mathrm{~S}: \mathrm{Tb}^{3+}$ powder phosphors, J. Lumin. 72-74 (1997) 772.

[19] Y. Glinec et al., Absolute calibration for a broad range single shot electron spectrometer, Rev. Sci. Instrum. 77 (2006) 103301.

[20] A. Buck et al., Absolute charge calibration of scintillating screens for relativistic electron detection, Rev. Sci. Instrum. 81 (2010) 033301. 
[21] K. Nakamura et al., Electron beam charge diagnostics for laser plasma accelerators, Phys. Rev. Spec. Top.-Accel. Beams 14 (2011) 062801.

[22] K. Zeil et al., Absolute response of Fuji imaging plate detectors to picosecond-electron bunches, Rev. Sci. Instrum. 81 (2010) 013307.

[23] S. Masuda et al., Absolute calibration of an electron spectrometer using high energy electrons produced by the laser-plasma interaction, Rev. Sci. Instrum. 79 (2008) 083301.

[24] B. Hidding et al., Novel method for characterizing relativistic electron beams in a harsh laser-plasma environment, Rev. Sci. Instrum. 78 (2017) 083301.

[25] Y. C. Wu et al., Note: Absolute calibration of two DRZ phosphor screens using ultrashort electron bunch, Rev. Sci. Instrum. 83 (2012) 026101.

[26] G. E. Giakoumakis and D. M. Miliotis, Light angular distribution of fluorescent screens excited by x-rays, Phys. Med. Biol. 30 (1985) 21.

[27] J. B. Birks, D. Fry, L. Costrell, and K. Kaniah, The Theory and Practice of Scintillation Counting, Pergamon Press, Oxford (1964). 